\title{
El desastre ambiental que se convirtió en mercancía invaluable
}

La obra de María del Carmen Zetina aquí reseñada es un recuento magistral de la inacción de una sociedad que no quiere hacerse responsable de sus actos y termina convirtiendo un territorio que podría ser fuente de riqueza y bienestar en un desastre ambiental difícil de atender. La sociedad aludida está integrada por varias generaciones de pobladores, los funcionarios de Gobierno de todos los niveles, los académicos y los empresarios mineros. Toda proporción guardada, este recuento se asemeja al argumento de la película de Richard Fleischer, Cuando el destino nos alcance (Soylent Green), que relata el caos que produce la indiferencia humana ante un panorama de autodestrucción y negación de una realidad.

El planteamiento general de esta investigación mantiene a lo largo del texto la premisa de que los habitantes de dicho territorio, conocido como $\mathrm{La}$ Zacatecana, en Guadalupe, Zacatecas, no perciben

- ProfesorInvestigador de la Dirección Regional Noroeste del Departamento de Estudios Urbanos y Medio Ambiente de El Colegio de la Frontera Norte (Colef), México. ORCID: https://orcid.org/0000000I-9085-I375 gcordova@colef.mx el riesgo que corren, principalmente por contaminación derivada de metales pesados, entre ellos el mercurio, y que, por el contrario, comparten la fantasía de que viven en un territorio generoso y próspero.

María del Carmen Zetina Rodríguez (2016). El desastre ambiental que se convirtió en una mercancía invaluable. La Zacatecana en Guadalupe, Zacatecas. Ciudad de México: El Colegio de San Luis y Universidad Autónoma de Ciudad Juárez. 
A la autora le preocupan, entre otras cosas, cómo la lógica de las rutinas dentro del ejido y de las instancias estatales (gubernamentales) conduce hacia consecuencias no buscadas, de qué forma se vive rodeado de agua y suelos contaminados, y cómo los residentes recrearon la idea de que los metales pesados y las aguas negras no trajeron consecuencias negativas a la salud ni a las labores agrarias, todo lo cual constituye, según la autora, una realidad que aprendieron a manejar día a día.

La investigadora sostiene que en el imaginario colectivo persiste la idea de que

Nuestras tierras son un tesoro invaluable; son muy productivas, las podemos negociar con quien se ponga enfrente, por ejemplo, con el capital privado, que se muere de ganas por poseerlas; son inocuas, aunque muchos digan lo contrario; es una leyenda negra lo que se dice de este lugar; seguimos esperando a compañías para que exploten la presa, y el Gobierno nos apoya, él entiende nuestros anhelos. (pp. 23-24)

El texto se divide en seis capítulos, donde la autora, inteligentemente, parte de preguntas que invitan a leer hasta ver las respuestas a cada una de ellas. Desde el primer capítulo, donde se exponen los enfoques teóricos, la autora es enfática al señalar la miopía de los pobladores de La Zacatecana, que no hacen nada para atender un problema ambiental y de salud pública, mientras viven soñando que algún día tendrán la oportunidad de vender o negociar sus terrenos y solucionar su pobreza generalizada.

En el segundo capítulo, la autora nos regala, entre otras cosas, la historia económica del lugar, en especial de cómo se construyó la mercancía y cómo se contaminó la laguna del territorio. En el tercer capítulo, se abordan las condiciones sociales en el contexto del valor de la tierra, y se apunta al desarrollo humano de la población analizada; en este punto,

\section{2}


se revela una historia de pobreza, marginación y abandono por parte de las autoridades.

En el cuarto capítulo, se señalan los proyectos mineros, que alientan la esperanza de que el territorio pueda aliviar su enfermedad crónico-degenerativa por la intervención de autoridades federales y empresas, en un intento por sacar ganancia económica para todos $\mathrm{y}$, eventualmente, dejar el suelo en mejores condiciones para evitar el daño al ambiente y la salud. En el quinto y sexto capítulos, se remarca el desastre ambiental que escala el problema a un asunto internacional, así como las alternativas de remediación del suelo que han sido infructuosas por lo especiales que son los ejidatarios.

El libro compone un relato que alcanza a elevar el pensamiento del lector sobre la condición humana y las pocas capacidades que tenemos como país para tratar de abordar este tipo de problemáticas, que para nuestra desgracia no sólo ocurren en La Zacatecana, sino en cada vez más casos de desastres ambientales provocados, como los derrames constantes de petróleo en el Golfo de México, la muerte de miles de aves en la Presa de Silva, en Guanajuato (1994), la contaminación de la población por metales de la fundición en Torreón, documentada a partir de los años ochenta, y recientemente, el derrame de sustancias tóxicas al río Sonora por la minera de Buenavista del Cobre, perteneciente al gigante minero Grupo México. Estos accidentes confirman la poca atención dada en el país a la prevención, y la simulación generalizada de una realidad que se revela a través de ventanas publicitarias pero se extingue tan pronto pasa la emergencia.

En el primer capítulo, la autora señala:

A diferencia de otras regiones en donde los afectados por proyectos mineros o de extracción se movilizan o viven inconformes con este tipo 
de industrias, los ejidatarios de La Zacatecana esperan con entusiasmo la llegada de este tipo de empresas en sus tierras. (p. 23)

Para tratar de explicar la específica realidad social que el libro relata, la experimentada académica recurre al método hermenéutico y se familiariza con las formas de vida en que las actividades humanas de La Zacatecana se expresan. Para ello, parte del supuesto de que "un ser humano es un agente intencional cuyas actividades obedecen a razones y que es capaz, si se le pregunta, de abundar discursivamente sobre esas razones (lo que incluye mentir acerca de ellas)" (Giddens, 2016, p. 41).

Con base en este planteamiento, y como resultado de su investigación, la autora deja claro que la sociedad en cuestión se encuentra totalmente desorientada y presa de su egoísmo, que ha perdido capacidades, como la humildad, que le permitan reconocer una problemática que ahoga a un sector muy vulnerable de su propio grupo, y que necesita de la voluntad de sus propios integrantes para salvarse de su situación, sumamente grave. Por el lado institucional, el texto permite observar la incapacidad de las instituciones para hacer frente a un problema de la magnitud del abordado.

En esta investigación, resulta revelador partir de la ecología política, que se basa en el postmaterialismo en sociedades económicamente avanzadas, y del ecologismo popular, que propone una defensa contra el mercado y el Estado, donde en palabras de Joan Martínez Alier, el valor de un territorio se vincula a los significados construidos sobre él. En el caso del problema abordado en el libro de la Dra. Zetina, el significado de tesoro local le da al territorio el valor de cambio que alimenta la esperanza de sus pobladores de salir de la pobreza.

El texto también recurre a la economía ecológica, que mide el papel de los diferentes actores sociales en el manejo 
del medio ambiente. En especial, este enfoque sirve para ver las acciones que cada actor realiza para defender sus intereses, incluidas las del Estado, que actúa como facilitador de las empresas mineras (legal, institucional, gestor, solucionador de conflictos o mediador). En este contexto, las legislaciones dejan resquicios por donde pueden operar las empresas mineras, que mantienen estrategias para convencer a la población y coaccionar o reprimir a sus opositores, como las oenegés. Esto genera, como alguna vez señalara Arturo Escobar, movimientos sociales en defensa del territorio, redes, vínculos y actores con poder, en donde el territorio es un espacio multidimensional que incluye cultura, sociedad y economía.

Las distintas perspectivas que la autora adopta justifican que pensemos en una sociedad más atenta y participativa en los asuntos públicos, en respuesta a que el Estado y el mercado conspiran en su contra al no querer mejorar el medio ambiente y la salud humana en el territorio bajo estudio. Con ello, se advierte el poder que tiene el primero y la asimetría que hay en las relaciones sociales, representada, por ejemplo, en el asociativismo que ocurre entre Estado y grupos de presión económica, una forma de reproducción del capital alejada del interés general y muy permisiva en la atención de las externalidades, en este caso ambientales. Esto es señalado por la autora como desigualdad en la distribución de los riesgos, y genera temor y decisiones un tanto incoherentes por parte de los habitantes, que buscan con ansia mejorar sus vidas.

Sobre esto último, la autora es generosa y se detiene a explicar esta situación por medio de autores como Ulrich Beck y Mary Douglas, que han señalado la exigencia de búsqueda de riesgo emprendida por algunas sociedades. En La Zacatecana, incluso sobrevivir sobrelleva riesgo: sus habitantes se la juegan para proteger ciertos valores e intereses comunitarios, como señala la autora. 
En torno a dicho afán del riesgo, surge una nube de incertidumbre que envuelve esta investigación, y que se acompaña por la perspectiva de la estructuración de Giddens, un autor de gran valor para explicar cómo se construyó un imaginario colectivo que cuenta con una historia llena de mitos y leyendas. La autora parte de que los ejidatarios son los más interesados en que sus tierras sean explotadas por extraños. En ellos, hay lo que Giddens llama conciencia práctica, es decir, los actores locales son conscientes de lo que hacen.

Este último enfoque, muy avanzado, permite reconstruir una historia de las motivaciones que llevan al grueso de la población de La Zacatecana a aceptar no sólo la contaminación de su territorio por metales pesados liberados por la minería, sino la recepción de un sinfín de contaminantes a través de las aguas residuales o los vapores de las ladrilleras asentadas en el lugar.

Esta consciencia práctica es terreno fértil para que se haya desarrollado un escenario que no tiene sustento en la tierra, sino en la imaginación de todos los actores. Se aprecia que incluso el Gobierno y los empresarios mineros pueden avanzar en sus propósitos porque los propietarios del suelo no se quejan de ningún modo, víctimas de la ignorancia y la desorientación. Además, ellos mismos fomentan la explotación de sus tierras, sin importarles las consecuencias a la salud y al deterioro de sus recursos.

Por otro lado, el teorema de la dualidad de Giddens dice que "la constitución de agentes y la de estructuras no son dos conjuntos de fenómenos dados independientemente, no forman un dualismo[,] sino que representan una dualidad" (2016, p. 35). Así se explica que las conductas y actitudes de La Zacatecana no son meramente sacadas del imaginario de sus habitantes, sino que forman parte de un conjunto de factores interrelacionados que dan como resultado un asunto complejo en donde van influyendo en una trama sin

\section{6}


fin cuestiones políticas como la creación de ejidos después de la Revolución mexicana, la desaparición de la hacienda Trancoso, el paternalismo de los Gobiernos revolucionarios hacia el sector del campo, las expansiones urbanas de las ciudades de Zacatecas y Guadalupe, el interés de organismos internacionales, entre otros aspectos. Hay, como lo señala Giddens, "circunstancias más amplias que afectan su propio entorno" (2016, p. 241), y que la Dra. Zetina busca revelar desde muchas fuentes.

En resumen, desde el primer capítulo del libro quedan claras varias ideas sobre el objeto de esta investigación: 1) la certidumbre entre la población de que su tierra tiene un gran valor; 2) el aprendizaje de la población del fingimiento sobre problemas ambientales porque no desea conocer lo que no le es conveniente; 3) la opinión de otros actores, que confirma su certidumbre; 4) la posesión de las tierras como mercancía; 5) la creencia de que el valor que tienen sus tierras es la fuente de conflicto, lo que les confiere mucho poder; 6) el manejo de un discurso según el cual la población nunca ha sido afectada por la contaminación; y 7) la existencia de una controversia entre actores académicos, por lo que la ciencia se encuentra en entredicho.

En el capítulo dos, se expone una reconstrucción de hechos y discursos para ejecutar una biografía económica que parte del descubrimiento de un tesoro de metales preciosos debajo de la siembras en las inmediaciones de la laguna, esto sucedido en la época en que operaba eficientemente la hacienda Trancoso. Los habitantes del lugar fueron testigos de cómo un particular, Alberto Delissalde, se hizo rico: "sacaban lingotes fundidos" (p. 81); esto alimentó la codicia por esas tierras, y gracias a un litigio, aunque prolongado -de la revolución a los años cincuenta-, los pobladores del lugar pudieron también hacerse ricos cuando consiguieron que la empresa minera Santa Teresa, explotadora de los jales, les pagaran una cuantiosa renta. 
Las referencias de la riqueza de los jales de la laguna la expone de alguna manera uno de los entrevistados de la Dra. Zetina, Santos Basurto, quien vio la casa de Delissalde en la ciudad de Zacatecas y a todo el pueblo contaba la opulencia en que vivía el empresario minero. Esto fue creando una leyenda que ha marcado la consciencia de los pobladores de La Zacatecana y de otros tantos actores que después tuvieron antojo por ese suculento pastel. Entonces, se habla del oro y la plata, el territorio como fuente de riqueza y la confianza de los pobladores en sus recursos naturales y el consumo de estos -como los peces de la laguna-. No se mencionan la contaminación generalizada por aguas negras ni los 300 a 500 gramos de mercurio vertidos por tonelada de metal extraído.

En el capítulo tres, se relata una historia de empobrecimiento que ha cursado generaciones, desde la existencia bajo el amparo de la hacienda hasta nuestros días. Se aprecia, en efecto, una alta vulnerabilidad social por el bajo ingreso, la falta de empleo, la migración de muchos hacia el otro lado, la amenaza de la expansión urbana y la mala calidad de vida por la contaminación. Cerca del 50\% de las familias recibe apoyo federal (programa Oportunidades), y los jefes de hogar generalmente alternan sus actividades productivas entre la albañilería y la agricultura, con pocos apoyos de los Gobiernos.

Es muy relevante en este capítulo el trabajo etnográfico que se hace de la comunidad de La Zacatecana. Por ejemplo, los testimonios de los Basurto y los Bernal son fundamentales para reconstruir la historia de muchas familias (si no es que de la mayoría) de esa población y las aledañas. Dichas experiencias relatadas por la autora enriquecen la parte empírica de su trabajo, lo que sin duda da buenos dividendos a la hora de concluirlo.

En el capítulo cuatro, se exponen las controversias suscitadas entre autoridades, ejidatarios, empresarios y aca-

\section{8}


démicos en torno al territorio de La Zacatecana. Una vez reforzadas las instituciones de corte ambiental en el país y establecidas leyes, reglamentos y normas de gran envergadura en materia ambiental, el Estado reacciona ante un problema que es alentado por empresarios mineros con el cuento de la remediación del sitio. Hubo una "controversia ambiental" (p. 136) que se hizo pública a través de los medios de comunicación, y en la que apareció Minera Orca como la única interesada en la remediación.

El libro retrata cómo en dicha controversia ambiental se magnificó públicamente la mortandad de peces por el agua asolvada en la laguna. La Comisión Nacional del Agua (Conagua) le otorgó un permiso a Orca en el año 2005, ya que la empresa presentó una manifestación de impacto ambiental, como lo consigna el artículo 28 de la Ley General del Equilibrio Ecológico y Protección al Ambiente del país. El discurso de la Secretaría de Medio Ambiente y Recursos Naturales fue el de "matar dos pájaros de un tiro" (p. 146): se obtenía metal y se remediaba el problema ambiental en el sitio. Al final, pesó la desconfianza en las instituciones por su aparente apoyo a la iniciativa privada. Al respecto, es interesante la intervención de la Comisión de Cooperación de Norteamérica (CCA), creada a partir del Tratado de Libre Comercio de América del Norte (TLCAN), que funcionó como un tercero para dirimir la controversia.

En el capítulo quinto, se hace un recuento del desastre ambiental y del escalamiento del problema de lo local a lo internacional. La presencia de la CCA y la Organización Mundial de la Salud (oms) es signo del intento de poner fin a una serie de conflictos heredados de la región de Norteamérica y de los nulos resultados de las autoridades ante problemas ambientales complejos como este. Son inquietantes las versiones de que la población de La Zacatecana sí es afectada en su salud, como atestigua el académico Arturo López, de la Universidad Autónoma de Zacatecas, a quien 
el libro recupera por su establecimiento del vínculo entre el mercurio liberado al ambiente y casos de osteoporosis en la población (p. 174), esto a pesar de que el dictamen del Mexican Group on Mercury no fuera concluyente al respecto.

En el sexto capítulo, se hace un ensayo de la remediación ambiental emprendida a pesar de la oposición de los principales actores del territorio: los ejidatarios, quienes no quieren experimentos de remediación ni donación de tierra para dicho fin, y buscan más bien un arreglo para mantener su fantasía. Actúan a la defensiva con funcionarios y empresarios (p. 205). Es el caso de Manuel Bernal, quien puso un precio alto a su predio en las negociaciones porque cree que en él hay un tesoro.

Finalmente, hay que señalar, a manera de conclusión, que el trabajo de la Dra. Zetina es muy relevante y abona indiscutiblemente a la comprensión de un fenómeno social de negación de una realidad que ya alcanzó a la población, que la ha dañado y lo sigue haciendo, aunque esta no le quiera poner punto final por desconfianza, justificada, hacia otros actores que podrían aprovecharse de la comunidad, que es pobre pero con dignidad.

Giddens, A. (2016). La constitución de la sociedad. Bases para la teoría de la estructuración. Buenos Aires:Amorrortu. 